\title{
University Teachers' Professional Title Evaluation Prediction Mode Based on LVQ Neural Network
}

\author{
Jigang Zhang ${ }^{1, ~ a, N a ~ L i a n g ~}{ }^{2, b}$ \\ ${ }^{1}$ Department of Mathematics HuBei University Of Science And Technology, HuBei Xianning, China \\ 2 Department of Mathematics HuBei University Of Science And Technology, HuBei Xianning, China \\ axnzhjg@163.com, b965352379@qq.com
}

Keywords: university teachers' professional title evaluation,LVQ neural network

\begin{abstract}
At present, university teachers' professional title evaluation is still mainly based on the qualitative analysis in our country, having their limitation, for example low efficiency, tedious process, complex operation and so on. On the basis of the Learning vector quantization neural network(LVQ), a new model for university teachers' professional title evaluation is proposed. The paper has constructed the nonlinear mapping relationship between evaluation materials and promotion to evaluate university teachers' professional title, using LVQ's advantages of simple network structure, self- learning, self- organization, and nonlinear classification processing capacity. The experiment results show that the model is effective, which gives a new way for the evaluation on university teachers' professional title.
\end{abstract}

\section{Introduction}

At the present stage, university teachers' professional title evaluation is still mainly based on the qualitative analysis in our country. Because of complex actual operation and inevitable subjectivity, the result was not accurate enough. How to quickly establish a objective, efficient model to accurately evaluate the promotion of teachers' professional title is an important problem for universities.

Considering the complex nonlinear relations among the teachers' title appraisal indicators, the artificial neural network is the only method that enables nonlinear adaptive information processing in all methods, In recent years it has been introduced to evaluate prediction. BP network is the most commonly used, whose weaknesses is likely to fall into a local minimum, can not guarantee the global minimum, using a nonlinear optimization method based on gradient descent [1,2].Although some other optimization strategy, for example genetic algorithm, simulated annealing algorithm and so on, can find the global minimum, but there are a very large amount of computation and the efficiency problem sometimes. The advantage of LVQ neural network is that input variables do not need to be normalized, normalized, the pattern recognition are realized only by directly calculating the distance between input variables and the competitive layer. This paper attempts to use LVQ neural network to predict university teachers' professional title evaluation based on the real data of 46 teachers of an university in 2012.

\section{LVQ neural network model}

LVQ neural network is a learning algorithm to train the competitive layer under supervision.It has the advantages of simple network structure, completing the very complex classification only through the interaction of internal unit. It has overcome the weakness of the lack of information 
classification of self-organizing network with unsupervised learning algorithm. It has been widely used in pattern recognition and optimization.

The LVQ network model $[3,4,5]$. is shown in Figure 1. It consists of three layers neurons, namely the input layer, the hidden layer and output layer. The input layer is fully connected to the hidden layer, in which the connection weights is reference vector, and the hidden layer is partially connected to the output layer, in which the connection weights is fixed to 1 .

During the training of the network, the connection weights between has been adjusted gradually to the clustering center. When an input sample is sent to the LVQ network, the competitive layer neuron of reference vector closest approach to the input sample wins the competition for excitation, then it's output is " 1 ", the other the competitive layer neuron output is " 0 ". The output of the output layer neurons connected with the winning neuron group is "1", providing the current input samples the pattern class, and other output is"0". The class received by competitive layer learning is called the subclass, and the class received by output layer learning is called the goal class.

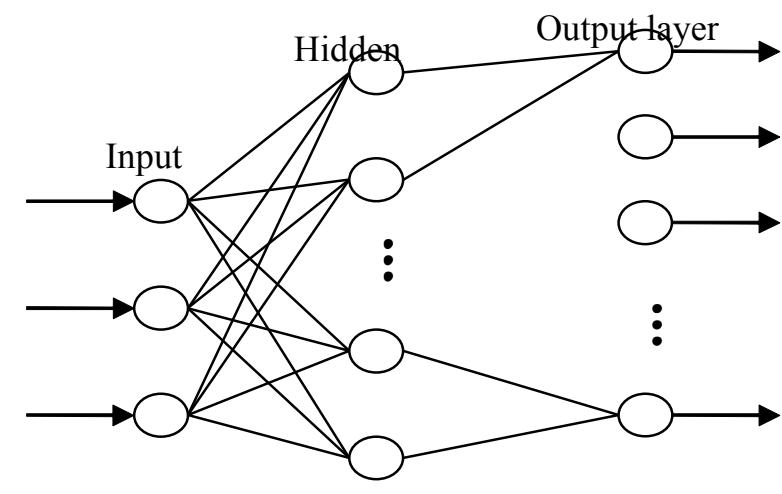

Figure. 1 LVQ network structure

\section{University teachers' professional title evaluation prediction model based on the LVQ network}

The professor titles evaluation's prediction indexes and sample data. According to the professor titles criterion of some University over the years, the acquisition of professor titles is to be assessed synthetically by considering the thesis quality or quantity, science research achievements award, scientific research projects, etc. In the light of 46 teachers having undertaken totally scientific research projects, and individually few of them wining science research achievements award, the author put forward an evaluating index system: SCI/EI numbers $x_{1}$,al core journals numbers $x_{2}, \mathrm{a} 2$ core journals numbers $x_{3}, \mathrm{a} 3$ core journals numbers $x_{4}$, noncore journals numbers $x_{5}$, total number $x_{6}$. The original data is shown in table 1 .

Table. 1 the original data

\begin{tabular}{|c|c|c|c|c|c|c|c|c|c|c|c|c|c|}
\hline number & $x_{1}$ & $x_{2}$ & $x_{3}$ & $x_{4}$ & $x_{5}$ & $x_{6}$ & number & $x_{1}$ & $x_{2}$ & $x_{3}$ & $x_{4}$ & $x_{5}$ & $x_{6}$ \\
\hline 1 & 3 & 0 & 2 & 0 & 23 & 28 & 24 & 0 & 0 & 2 & 1 & 15 & 18 \\
\hline 2 & 0 & 0 & 1 & 2 & 12 & 15 & 25 & 4 & 0 & 0 & 6 & 7 & 17 \\
\hline 3 & 3 & 1 & 0 & 1 & 4 & 9 & 26 & 0 & 0 & 1 & 0 & 9 & 10 \\
\hline 4 & 2 & 0 & 14 & 2 & 0 & 18 & 27 & 0 & 0 & 5 & 0 & 2 & 7 \\
\hline 5 & 0 & 0 & 4 & 10 & 3 & 17 & 28 & 0 & 0 & 3 & 0 & 10 & 13 \\
\hline
\end{tabular}




\begin{tabular}{|c|c|c|c|c|c|c|c|c|c|c|c|c|c|}
\hline 6 & 0 & 1 & 9 & 6 & 3 & 19 & 29 & 0 & 0 & 6 & 2 & 14 & 22 \\
\hline 7 & 0 & 0 & 2 & 1 & 6 & 9 & 30 & 0 & 0 & 7 & 0 & 12 & 19 \\
\hline 8 & 0 & 0 & 1 & 2 & 9 & 11 & 31 & 0 & 0 & 8 & 1 & 9 & 18 \\
\hline 9 & 5 & 0 & 0 & 0 & 3 & 8 & 32 & 0 & 0 & 7 & 1 & 8 & 16 \\
\hline 10 & 0 & 0 & 1 & 3 & 19 & 23 & 33 & 0 & 0 & 7 & 1 & 16 & 24 \\
\hline 11 & 0 & 0 & 2 & 7 & 6 & 15 & 34 & 0 & 0 & 3 & 0 & 8 & 11 \\
\hline 12 & 3 & 0 & 0 & 2 & 9 & 14 & 35 & 0 & 0 & 7 & 6 & 11 & 24 \\
\hline 13 & 0 & 0 & 2 & 0 & 14 & 16 & 36 & 0 & 0 & 5 & 0 & 15 & 20 \\
\hline 14 & 0 & 1 & 1 & 0 & 12 & 14 & 37 & 1 & 0 & 5 & 0 & 9 & 15 \\
\hline 15 & 0 & 0 & 0 & 0 & 9 & 9 & 38 & 0 & 0 & 5 & 1 & 7 & 13 \\
\hline 16 & 0 & 0 & 2 & 0 & 15 & 17 & 39 & 0 & 0 & 4 & 0 & 9 & 13 \\
\hline 17 & 0 & 0 & 4 & 0 & 18 & 22 & 40 & 0 & 1 & 5 & 7 & 0 & 13 \\
\hline 18 & 0 & 0 & 4 & 0 & 11 & 15 & 41 & 0 & 0 & 1 & 3 & 3 & 7 \\
\hline 19 & 2 & 0 & 1 & 0 & 9 & 12 & 42 & 0 & 1 & 8 & 0 & 3 & 12 \\
\hline 20 & 4 & 0 & 2 & 3 & 6 & 15 & 43 & 0 & 0 & 1 & 2 & 3 & 6 \\
\hline 21 & 1 & 0 & 5 & 0 & 0 & 6 & 44 & 2 & 0 & 2 & 9 & 9 & 22 \\
\hline 22 & 4 & 0 & 3 & 0 & 4 & 11 & 45 & 4 & 0 & 4 & 0 & 6 & 14 \\
\hline 23 & 2 & 0 & 0 & 0 & 18 & 20 & 46 & 3 & 0 & 5 & 0 & 7 & 15 \\
\hline
\end{tabular}

In this paper, the sample data has been randomly divided into training samples and testing samples as shown in table 2. Because the elimination rate of professor titles evaluation is $40 \%$, and the best rate among the training samples and test sample is $4: 1$, the paper has taken the first 37 groups as training samples(14 elimination and 23 promotion)to train the LVQ network to establish the teachers' professional title evaluation model, the last 9 groups as test sample (4 elimination and 5 promotion) to estimate the prediction accuracy of the established model.

Table. 2 sample grouping

\begin{tabular}{|c|c|c|c|}
\hline & promotion & elimination & total \\
\hline training samples & 23 & 14 & 37 \\
\hline test sample & 5 & 4 & 9 \\
\hline total & 28 & 18 & 46 \\
\hline
\end{tabular}

The LVQ network model of professional title evaluation. The paper has established the LVQ network model with MATLAB neural network toolbox as a platform. Based on the previous analysis, the order of training samples matrix" $P$ train" is $6 \times 37$, and the order of testing samples matrix" $P$ test" is $6 \times 9$. Because one of the advantage of LVQ neural network is that input variables do not need to be normalized and normalized,the training samples matrix" $\mathrm{P}_{\text {_train" }}$ are the first 37 groups in Table 1, and testing samples are the last 9 groups.

The input vectors are divided into two different classes, using the number 1 as the professional title promotion and the number 2 as the elimination. Class vector" TC" is converted into goal vector" $T$ " by the function " ind2vec ". Because the classification performance of network is affected by the number of competition layer neuron, the number of competition layer neuron is selected through continuous experiment. After experimenting many times, the number has been selected 5.

The function " newlvq "is used to create a LVQ network ,where the learning rate and learning algorithms are set to the default values. net $=$ newlvq $(\min \max (P), 5,[0.60 .4])$; 
The function " train "is used to train the LVQ network, where the training step and the training error are set to the default values. net $=\operatorname{train}($ net $, P, T)$;

The function " sim "is used to simulation and prediction of the trained network, checking whether the correct classification. Simulation results showed that the LVQ network has high classification precision, suggesting that university teachers' professional title evaluation prediction model based on the LVQ network has been established.

The test sample " P_test "are predicted to check prediction accuracy of the LVQ model

$$
\begin{aligned}
& y=\operatorname{sim}\left(\text { net }, p_{-} \text {test }\right) \\
& y c=\operatorname{vec} 2 \operatorname{ind}(y) ;
\end{aligned}
$$

The output results are 221212111 , as shown in Table 3

Table .3 LVQ neural network prediction results and expert review

\begin{tabular}{|l|l|l|l|l|l|l|l|}
\hline Number & expert & LVQ & prediction & Number & expert & LVQ & prediction \\
\hline 38 & elimination & 2 & true & 43 & elimination & 2 & true \\
\hline 39 & elimination & 2 & true & 44 & promotion & 1 & true \\
\hline 40 & promotion & 1 & true & 45 & promotion & 1 & true \\
\hline 41 & elimination & 2 & true & 46 & promotion & 1 & true \\
\hline 42 & promotion & 1 & true & & & & \\
\hline
\end{tabular}

Table 3 showed that the prediction accuracy of the LVQ model has been reached 100 percent, Therefore, the LVQ model has a good classification function. So, university teachers' professional title evaluation prediction model based on the LVQ network has been constructed successfully.

\section{Summary}

The paper has constructed university teachers' professional title evaluation prediction model based on the LVQ network, and has made an empirical analysis of the real data of 46 teachers of an university in 2012, and has achieved high classification accuracy through the LVQ network training, simulation and test. The results show that university teachers' professional title evaluation prediction model based on the LVQ network can effectively predict, and the model may offer valuable reference information to university teachers' professional title evaluation, at the same time has verified that pattern recognition function of the LVQ network model is very good.

\section{References}

[1] Ji-Gang Zhang, Na Liang, Taxation Forecasting Model Based on ELman Neural Network,Statistics and decision, Commun 6 (2007)13-14.

[2]Fecit Science and Technology Research Center,Neural Network and Matlab Application, third ed,Publishing House of Electronics Industry, Beijing, 2005,pp.

[3]Pingfan Yan,Changshui, Zhang,Artificial neural network and Simulated evolutionary computation, fifth ed, Publishing House of Tsinghua University, Beijing,2002,pp.

[4]Na Liang, JiGang Zhang, The Estimate of Teaching Quality Based on SOM Neural Networks,Journal of HaiNan University, Commun 2(2012)144-146.

[5]Na Liang, JiGang Zhang, College graduate degree evaluation Forecasting Based on LVQ network [J] Gansu Science, Commun 3(2012)157-158 